\title{
Emergence and spectral-weight transfer of electronic states in the Hubbard ladder
}

\author{
Masanori Kohno* \\ National Institute for Materials Science, Tsukuba 305-0003, Japan
}

(Received 5 September 2019; revised manuscript received 3 December 2019; published 27 December 2019)

\begin{abstract}
The number of electronic bands is usually considered invariant regardless of the electron density in a band picture. However, in interacting systems, the spectral-weight distribution generally changes depending on the electron density, and electronic states can even emerge or disappear as the electron density changes. Here, to clarify how electronic states emerge and become dominant as the electron density changes, the spectral function of the Hubbard ladder with strong repulsion and strong intrarung hopping is studied using the non-Abelian dynamical density-matrix renormalization-group method. A mode emerging in the low-electron-density limit gains spectral weight as the electron density increases and governs the dimer Mott physics at quarter-filling. In contrast, the antibonding band, which is dominant in the low-electron-density regime, loses spectral weight and disappears at the Mott transition at half-filling, exhibiting the momentum-shifted magnetic dispersion relation in the small-doping limit. This paper identifies the origin of the electronic states responsible for the Mott transition and brings a new perspective to electronic bands by revealing the overall nature of electronic states over a wide energy and electron-density regime.
\end{abstract}

DOI: 10.1103/PhysRevB.100.235143

\section{INTRODUCTION}

In band theory, an electron is assumed to hop from one atomic orbital to another in an effective periodic potential, forming a band [1]; the number of bands is considered essentially determined by the number of atomic orbitals in a unit cell, which does not change with the electron density. In Fermi-liquid theory, electronic excitations other than the quasiparticle band are regarded as incoherent [2]; the incoherent excitations are usually considered almost featureless and unimportant regardless of the electron density.

However, in interacting systems, some electronic excitations generally become dominant among many excited states, and the number of dominant modes can change depending on the electron density. Electronic excitations away from the Fermi level can also become dominant and exhibit significant characteristics. Thus, revealing the overall nature of electronic states over a wide energy and electron-density regime is important in the deeper understanding of the effects of strong electronic correlations. In particular, strong correlations significantly affect the electronic states near the Mott transition, which have attracted considerable attention in relation to hightemperature superconductivity [3-5].

In this paper, to clarify how electronic states emerge, change, and disappear as the electron density changes in strongly correlated systems, the spectral function of the Hubbard ladder, which is one of the simplest models containing

\footnotetext{
*kohno.masanori@nims.go.jp
}

Published by the American Physical Society under the terms of the Creative Commons Attribution 4.0 International license. Further distribution of this work must maintain attribution to the author(s) and the published article's title, journal citation, and DOI. the essence of electronic correlations, is investigated in the regime of strong Coulomb repulsion and strong intrarung hopping. The qualitative features of the results would be generally true for coupled dimer systems, such as the dimer Mott insulators of molecular solids [6,7] regardless of the lattice structure or dimensionality as long as the Coulomb repulsion and intradimer hopping are much stronger than the interdimer hopping. In particular, the perturbative arguments shown in this paper can be straightforwardly extended to bilayer systems.

The main features we focus on in this paper are the (1) emergent electronic states in the low-electron-density regime [Sec. V], (2) spectral-weight transfer from the dominant modes to the emergent modes, which makes the emergent modes dominant, whereas the dominant modes significantly lose spectral weight as the electron density increases to halffilling [Sec. III], (3) dimer Mott gap at quarter-filling, whose value is significantly limited by the intrarung hopping in the strong-Coulomb-repulsion regime [Sec. VI], and (4) emergent electronic states upon doping a Mott insulator by which the Mott transition is characterized [Sec. VIII]. The above features are contrasted with conventional views, such as a band picture.

\section{MODEL AND METHOD}

We consider the Hubbard ladder defined by the following Hamiltonian:

$$
\begin{aligned}
\mathcal{H}= & -t_{\|} \sum_{i, \alpha, \sigma}\left(c_{i, \sigma}^{\alpha \dagger} c_{i+1, \sigma}^{\alpha}+\text { H.c. }\right)-t_{\perp} \sum_{i, \sigma}\left(c_{i, \sigma}^{1 \dagger} c_{i, \sigma}^{2}+\text { H.c. }\right) \\
& +U \sum_{i, \alpha} n_{i, \uparrow}^{\alpha} n_{i, \downarrow}^{\alpha}-\mu \sum_{i, \alpha, \sigma} n_{i, \sigma}^{\alpha}
\end{aligned}
$$


where $c_{i, \sigma}^{\alpha}$ and $n_{i, \sigma}^{\alpha}$, respectively, denote the annihilation and number operators of an electron with spin $\sigma(=\uparrow, \downarrow)$ at the site of leg $\alpha(=1,2)$ and rung $i$. Hereafter, the number of sites in a leg, total number of sites, and number of electrons are denoted by $L, N_{\mathrm{s}}(=2 L)$, and $N_{\mathrm{e}}$, respectively. The doping concentration $\delta$ is defined as $\delta=1-n$, where $n$ denotes the electron density $\left(n=N_{\mathrm{e}} / N_{\mathrm{s}}\right)$.

The single-particle spectral function $A(\boldsymbol{k}, \omega)$ and the dynamical spin structure factor $S(\boldsymbol{k}, \omega)$ are defined as

$$
\begin{aligned}
& A(\boldsymbol{k}, \omega)= \begin{cases}\frac{1}{2} \sum_{l, \sigma}\left|\left\langle l\left|c_{\boldsymbol{k}, \sigma}^{\dagger}\right| \mathrm{GS}\right\rangle\right|^{2} \delta\left(\omega-\varepsilon_{l}\right) & \text { for } \omega>0, \\
\frac{1}{2} \sum_{l, \sigma}\left|\left\langle l\left|c_{\boldsymbol{k}, \sigma}\right| \mathrm{GS}\right\rangle\right|^{2} \delta\left(\omega+\varepsilon_{l}\right) & \text { for } \omega<0,\end{cases} \\
& S(\boldsymbol{k}, \omega)=\frac{1}{3} \sum_{l, \gamma}\left|\left\langle l\left|S_{\boldsymbol{k}}^{\gamma}\right| \mathrm{GS}\right\rangle\right|^{2} \delta\left(\omega-\varepsilon_{l}\right),
\end{aligned}
$$

where $\varepsilon_{l}$ represents the excitation energy of the eigenstate $|l\rangle$ from the ground state $|\mathrm{GS}\rangle$. Here, $c_{\boldsymbol{k}, \sigma}^{\dagger}$ and $S_{\boldsymbol{k}}^{\gamma}$ denote the Fourier transform of $c_{i, \sigma}^{\dagger}$ and that of the $\gamma(=x, y, z)$ component of the spin operator $\boldsymbol{S}_{i}$, respectively. For ladders, $\boldsymbol{k}=\left(k_{\|}, k_{\perp}\right)$, where $k_{\|}$and $k_{\perp}$ denote the momenta in the leg and rung directions, respectively. Because $\left\{c_{\boldsymbol{k}, \sigma}, c_{\boldsymbol{k}, \sigma}^{\dagger}\right\}=$ $1, A(\boldsymbol{k}, \omega)$ satisfies the following sum rule at each $\boldsymbol{k}$ :

$$
\int_{-\infty}^{\infty} d \omega A(\boldsymbol{k}, \omega)=1 .
$$

We consider the case of $0 \leqslant n \leqslant 1$ without loss of generality because $A(\boldsymbol{k}, \omega)$ for $1<n \leqslant 2$ can be obtained as $A(\boldsymbol{k}+\boldsymbol{\pi},-\omega)$ at the electron density $2-n$ by using the particle-hole transformation. The Hubbard ladder has been studied primarily on the ground-state properties [8-12], spin and charge excitations [13-15], spectral function around halffilling $[10,15,16]$, charge and photodynamics $[17,18]$, and ferromagnetism [19-21]. In this paper, to clarify the evolution of electronic states as a function of the electron density, we investigate the spectral function in the overall electrondensity regime primarily for $U \gg t_{\perp} \gg t_{\|}>0\left(U t_{\|} / t_{\perp}^{2}\right.$ is not too large for the ground state to have spin 0 or $1 / 2$ $[19,20])$ based on the numerical results for $U / t_{\|}=16$ and $t_{\perp} / t_{\|}=2$ obtained using the non-Abelian dynamical densitymatrix renormalization-group (DDMRG) method [5,22-27]. The DDMRG calculations were performed on a 120 -site cluster under open boundary conditions with 240 states retained for the density matrix. The truncation errors are negligibly small in the scales used for the figures in this paper.

\section{OVERALL SPECTRAL FEATURES}

The spectral-weight distributions of electronic states from the low-electron-density regime $(n \approx 0.083)$ to half-filling $(n=1)$ are shown in Fig. 1 for $k_{\perp}=0$ [Figs. 1(a)-1(h)] and $k_{\perp}=\pi$ [Figs. 1(i)-1(p)]. One might naively expect that the dominant modes in the low-electron-density regime $[0 \lesssim$ $\omega / t_{\|} \lesssim 4$ in Fig. 1(a); $4 \lesssim \omega / t_{\|} \lesssim 8$ in Fig. 1(i)] continuously deform into those at half-filling $\left[-3 \lesssim \omega / t_{\|} \lesssim 0\right.$ in Fig. 1(h); $11 \lesssim \omega / t_{\|} \lesssim 14$ in Fig. 1(p)] as the electron density increases. However, they are different in origin.

The dominant mode in the low-electron-density regime for $k_{\perp}=0$ [Fig. 1(a)] gradually loses spectral weight with the electron density [Figs. 1(b) and 1(c)]. At quarter-filling
( $n=1 / 2$ ), this mode is located below $\omega=0$, separated by a small gap from the mode above $\omega=0$ [Figs. 1(d) and 2(d)]. The spectral weight further decreases and almost disappears at half-filling [Figs. 1(e)-1(h)].

As for $k_{\perp}=\pi$, the dominant mode in the low-electrondensity regime [Fig. 1(i)] gradually loses spectral weight with the electron density [Figs. 1(j)-1(o)] and completely disappears at half-filling [Fig. 1(p)].

Instead, because of the sum rule [Eq. (3)], emergent modes in the low-electron-density regime [Figs. 1(a) and 1(i)] gradually gain spectral weight [Figs. 1(b)-1(g), 1(j)-1(o)], and become dominant at half-filling [Figs. 1(h) and 1(p)]. Thus, a significant amount of the spectral weight transfers from the dominant modes to the emergent modes as the electron density increases.

It should be noted that not only the high-energy modes of $O(U)$, but also an intermediate-energy mode emerges $\left[\omega / t_{\|} \approx\right.$ 7 and $k_{\|} \approx \pi$ in Fig. 1(a)], which is separated from the low-energy bonding band by an energy gap, and becomes the most dominant at half-filling for $k_{\perp}=0\left[-3 \lesssim \omega / t_{\|} \lesssim 0\right.$ in Fig. 1(h)].

These features are due to strong electronic correlations and contrast with a rigid-band picture in which the bonding and antibonding bands remain dominant regardless of the electron density.

\section{ZERO ELECTRON DENSITY}

To understand the nature of such complicated spectral features, we consider the properties of characteristic modes from the low-electron-density side. At zero electron density $(n=0)$, the electron-addition spectra show the noninteracting dispersion relations because the interaction term does not work with the added electron. Thus, the Hamiltonian $(U=0)$ can be diagonalized in the momentum space as

$$
\mathcal{H}_{0}=\sum_{k, \sigma} \epsilon_{k}^{0} c_{k, \sigma}^{\dagger} c_{k, \sigma} .
$$

The noninteracting dispersion relation $\epsilon_{\boldsymbol{k}}^{0}$ is obtained as

$$
\epsilon_{k}^{0}=-2 t_{\|} \cos k_{\|}-t_{\perp} \cos k_{\perp}-\mu,
$$

where $k_{\perp}=0$ for the bonding band and $k_{\perp}=\pi$ for the antibonding band (solid black curves in Fig. 3). In the lowelectron-density limit $(n \rightarrow 0), \mu \rightarrow-2 t_{\|}-t_{\perp}$.

\section{LOW ELECTRON DENSITY}

\section{A. High-energy emergent modes}

In addition to the dominant modes originating from the noninteracting bonding and antibonding bands [Eq. (5)], small spectral weights emerge when the electron density becomes nonzero [Figs. 1(a) and 1(i)]. The emergent modes at high energies of $O(U)$ can be regarded as the upper Hubbard bands. To capture their characteristics, we consider a two-electron system. The energy of a spin-singlet eigenstate $\epsilon_{\boldsymbol{k}}$ is generally obtained as a solution of the following equation [28]:

$$
1=\frac{U}{N_{\mathrm{s}}} \sum_{p} \frac{1}{\epsilon_{k}-\epsilon_{k-p}^{0}-\epsilon_{p}^{0}} .
$$




$$
k_{\perp}=0
$$
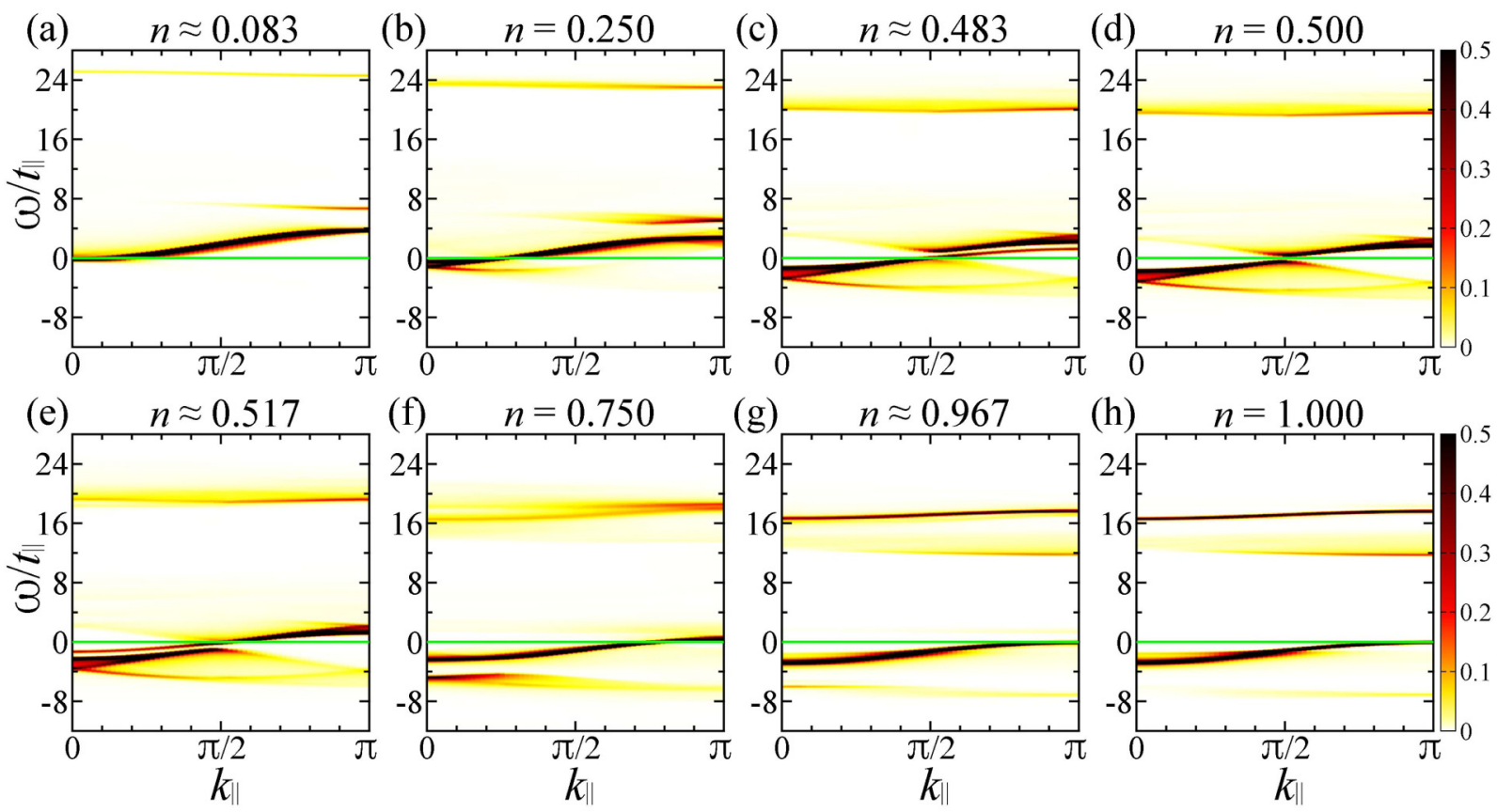

$$
k_{\perp}=\pi
$$
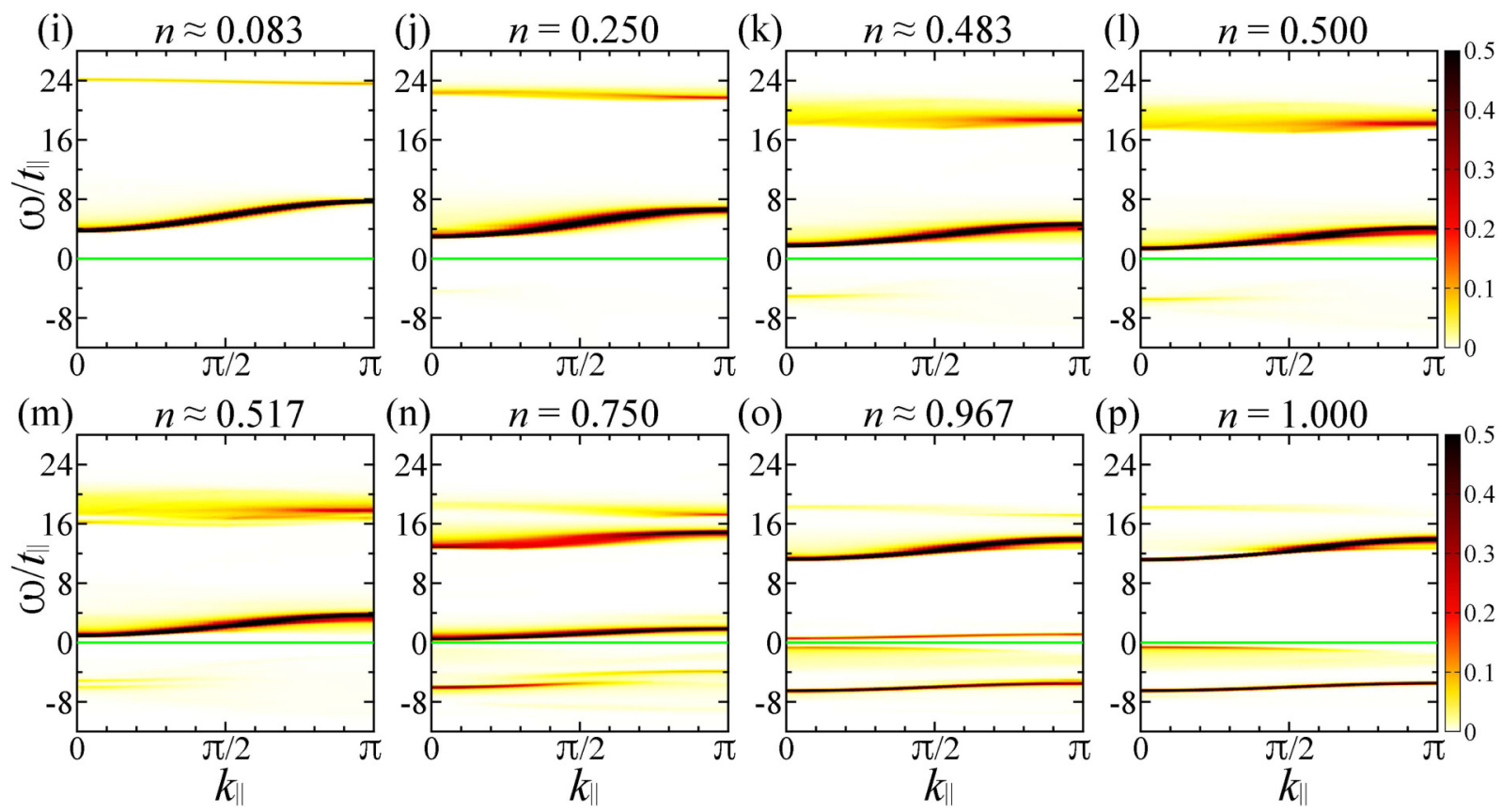

FIG. 1. $A(\boldsymbol{k}, \omega) t_{\|}$for $[(\mathrm{a})-(\mathrm{h})] k_{\perp}=0$ and [(i)-(p)] $\pi$ at [(a), (i) $n \approx 0.083,[(\mathrm{~b}),(\mathrm{j})] 0.25,[(\mathrm{c}),(\mathrm{k})] 0.483,[(\mathrm{~d}),(\mathrm{l})] 0.5,[(\mathrm{e}),(\mathrm{m})] 0.517$, $[(\mathrm{f}),(\mathrm{n})] 0.75,[(\mathrm{~g}),(\mathrm{o})] 0.967$, and $[(\mathrm{h}),(\mathrm{p})] 1$ for $U / t_{\|}=16$ and $t_{\perp} / t_{\|}=2$ obtained using the non-Abelian DDMRG method. The green lines indicate $\omega=0$. Gaussian broadening with a standard deviation of $0.1 t_{\|}$has been used.

By expanding Eq. (6) in powers of $U$, the dispersion relations of the high-energy modes for $U \gg t_{\perp}, t_{\|}$can be obtained as

$$
\begin{aligned}
& \epsilon_{\left(k_{\|}, 0\right)}=U+2 t_{\perp}+4 t_{\|}+J_{\|}\left(\cos k_{\|}+1\right)+J_{\perp}, \\
& \epsilon_{\left(k_{\|}, \pi\right)}=U+2 t_{\perp}+4 t_{\|}+J_{\|}\left(\cos k_{\|}+1\right),
\end{aligned}
$$

up to $O\left(t_{\|}^{2} / U\right)$ and $O\left(t_{\perp}^{2} / U\right)$, where $J_{\|}=4 t_{\|}^{2} / U$ and $J_{\perp}=$ $4 t_{\perp}^{2} / U$ (dotted orange curves in Fig. 3).
The above results can also be explained in terms of the modes of double occupancy. We define $\left|\psi^{+}\left(k_{\|}\right)\right\rangle$and $\left|D^{-}\left(k_{\|}\right)\right\rangle$ for $k_{\perp}=0$ and $\pi$, respectively, as

$$
\left|X\left(k_{\|}\right)\right\rangle=\frac{1}{\sqrt{L}} \sum_{j=1}^{L} e^{i k_{\|} r_{j}}|X\rangle_{j} \prod_{l \neq j}^{L}|0\rangle_{l},
$$

where $r_{j}$ denotes the coordinate of rung $j$ in the leg direction, and $X$ represents $\psi^{+}$and $D^{-}$. Here, $\left|\psi^{+}\right\rangle_{j},\left|D^{-}\right\rangle_{j}$, and $|0\rangle_{j}$ 

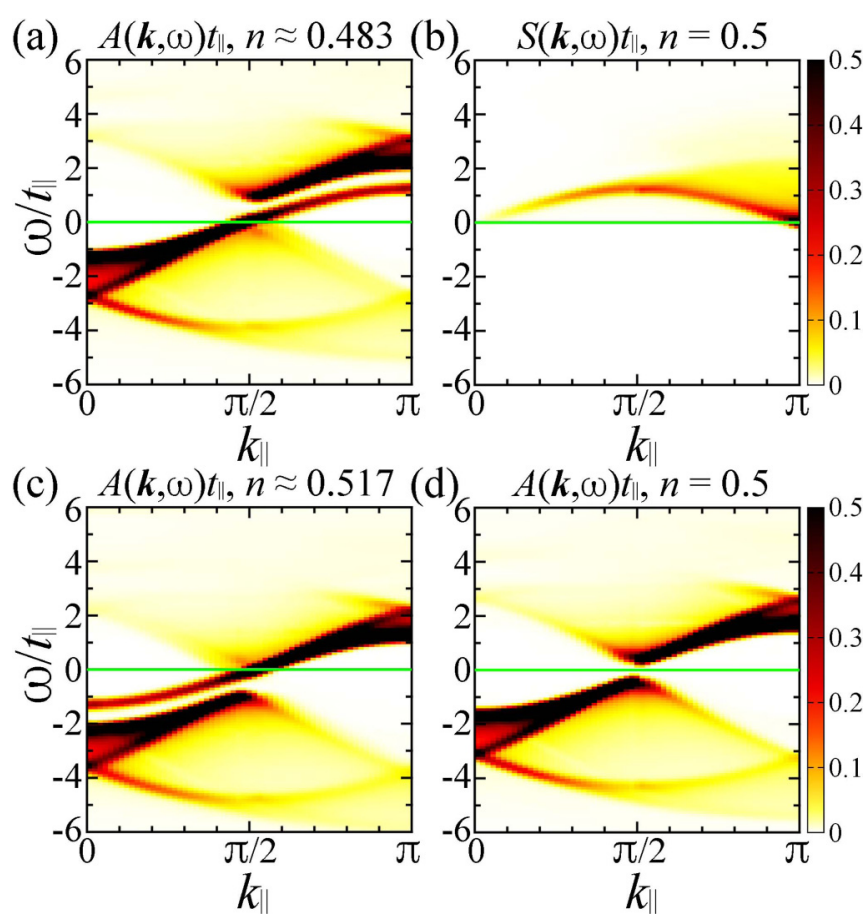

FIG. 2. (a), (c), (d) $A(\boldsymbol{k}, \omega) t_{\|}$for $k_{\perp}=0$ at [(a)] $n \approx 0.483$, [(c)] $n \approx 0.517$, and [(d)] $n=0.5$ for $U / t_{\|}=16$ and $t_{\perp} / t_{\|}=2$ obtained using the non-Abelian DDMRG method [closeup of Figs. 1(c), 1(e), and 1(d) near the Fermi level, respectively]. (b) $S(\boldsymbol{k}, \omega) t_{\|}$for $k_{\perp}=$ 0 at $n=0.5$ for $U / t_{\|}=16$ and $t_{\perp} / t_{\|}=2$ obtained using the nonAbelian DDMRG method. The green lines indicate $\omega=0$. Gaussian broadening with a standard deviation of $0.1 t_{\|}$has been used.

denote the eigenstates of the $j$ th rung defined in Table I. The effective hoppings of $\left|\psi^{+}\right\rangle$and $\left|D^{-}\right\rangle$are obtained using second-order perturbation theory with respect to $t_{\|}$as

$$
t_{\| \text {eff }}^{\psi^{+}}=-\frac{4 t_{\|}^{2}}{U}+\frac{2 t_{\|}^{2}}{\sqrt{U^{2}+16 t_{\perp}^{2}}}, \quad t_{\| \text {eff }}^{D^{-}}=-\frac{2 t_{\|}^{2}}{U},
$$
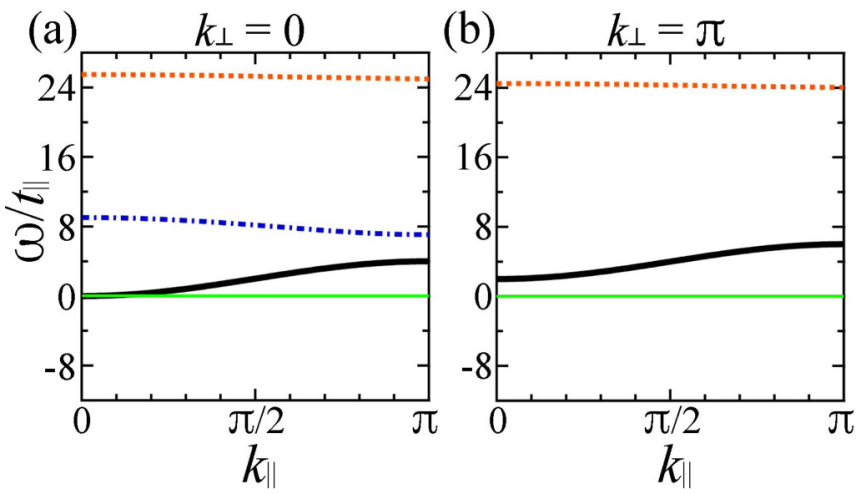

FIG. 3. Dispersion relation of electronic excitations in the lowelectron-density limit for $U / t_{\|}=16$ and $t_{\perp} / t_{\|}=2$ at $[(\mathrm{a})] k_{\perp}=0$ and $[(b)] \pi$. The dotted orange curves indicate the high-energy solutions of Eq. (6). The solid black curves indicate $\epsilon_{k}^{0}$ [Eq. (5)]. The dashed-dotted blue curve in (a) indicates the high-energy solutions of Eq. (6) for the effective Hubbard chain with $U_{\text {eff }}^{-}=E_{-}+2 t_{\perp}$ [Eq. (14)]. The green lines indicate $\omega=0$.
TABLE I. Eigenstates and energies on a rung.

\begin{tabular}{ll}
\hline \hline Eigenstate & Energy \\
\hline$|0\rangle=|0,0\rangle$ & $E_{0}=0$ \\
$\left|B_{\sigma}\right\rangle=(|\sigma, 0\rangle+|0, \sigma\rangle) / \sqrt{2}$ & $E_{B}=-t_{\perp}-\mu$ \\
$\left|A_{\sigma}\right\rangle=(|\sigma, 0\rangle-|0, \sigma\rangle) / \sqrt{2}$ & $E_{A}=t_{\perp}-\mu$ \\
$\left|\psi^{+}\right\rangle=-\zeta-|S\rangle+\zeta_{+}\left|D^{+}\right\rangle$ & $E_{\psi^{+}}=E_{+}-2 \mu$ \\
$\left|\psi^{-}\right\rangle=\zeta+|S\rangle+\zeta_{-}\left|D^{+}\right\rangle$ & $E_{\psi^{-}}=E_{-}-2 \mu$ \\
$\left|T^{+}\right\rangle=|\uparrow, \uparrow\rangle$ & $E_{T^{+}}=-2 \mu$ \\
$\left|T^{-}\right\rangle=|\downarrow, \downarrow\rangle$ & $E_{T^{-}}=-2 \mu$ \\
$\left|T^{0}\right\rangle=(|\uparrow, \downarrow\rangle+|\downarrow, \uparrow\rangle) / \sqrt{2}$ & $E_{T^{0}}=-2 \mu$ \\
$\left|D^{-}\right\rangle$ & $E_{D^{-}}=U-2 \mu$ \\
$\left|G_{\sigma}\right\rangle=(|\sigma, \uparrow \downarrow\rangle+|\uparrow \downarrow, \sigma\rangle) / \sqrt{2}$ & $E_{G}=U+t_{\perp}-3 \mu$ \\
$\left|F_{\sigma}\right\rangle=(|\sigma, \uparrow \downarrow\rangle-|\uparrow \downarrow, \sigma\rangle) / \sqrt{2}$ & $E_{F}=U-t_{\perp}-3 \mu$ \\
$|W\rangle=|\uparrow \downarrow, \uparrow \downarrow\rangle$ & $E_{W}=2 U-4 \mu$ \\
\hline \hline
\end{tabular}

$|S\rangle=(|\uparrow, \downarrow\rangle-|\downarrow, \uparrow\rangle) / \sqrt{2},\left|D^{ \pm}\right\rangle=(|\uparrow \downarrow, 0\rangle \pm|0, \downarrow \uparrow\rangle) / \sqrt{2}$, $\zeta_{ \pm}=\sqrt{\frac{1}{2}\left(1 \pm U / \sqrt{U^{2}+16 t_{\perp}^{2}}\right)}, E_{ \pm}=\frac{U}{2} \pm \frac{\sqrt{U^{2}+16 t_{\perp}^{2}}}{2}$.

respectively, which reduce to

$$
t_{\| \mathrm{eff}}^{\psi^{+}} \approx t_{\| \mathrm{eff}}^{D^{-}} \approx-\frac{J_{\|}}{2}
$$

for $U \gg t_{\perp}$. By taking into account the bond energy between $\left|\psi^{+}\right\rangle$and $|0\rangle, \xi_{\psi^{+} 0}\left(=-t_{\| \text {eff }}^{\psi^{+}}\right)$, and that between $\left|D^{-}\right\rangle$and $|0\rangle, \quad \xi_{D^{-} 0}\left(=-t_{\| \text {eff }}^{D^{-}}\right)$, the energies of the high-energy modes are obtained as

$$
\begin{aligned}
& E_{\| \text {eff }}^{\psi^{+}} \approx J_{\|}\left(\cos k_{\|}+1\right)+E_{+}-2 \mu, \\
& E_{\| \text {eff }}^{D^{-}} \approx J_{\|}\left(\cos k_{\|}+1\right)+U-2 \mu,
\end{aligned}
$$

up to $O\left(t_{\|}^{2} / U\right)$ for $U \gg t_{\perp} \gg t_{\|}$. Here,

$$
E_{ \pm}=\frac{U}{2} \pm \frac{\sqrt{U^{2}+16 t_{\perp}^{2}}}{2}
$$

which reduces to

$$
E_{+} \approx U+J_{\perp}, \quad E_{-} \approx-J_{\perp}
$$

for $U \gg t_{\perp}$. By putting $\mu=-2 t_{\|}-t_{\perp}$ in the low-electrondensity limit (the ground-state energy of the one-electron system is zero), Eq. (11) reduces to Eq. (7) for $U \gg t_{\perp} \gg$ $t_{\|}$. This result implies that the high-energy modes in the low-electron-density limit can be interpreted as the modes of double occupancy [Eq. (8)] for $U \gg t_{\perp} \gg t_{\|}$.

\section{B. Intermediate-energy emergent mode}

As mentioned in Sec. III, not only the high-energy modes of $O(U)$, but also an intermediate-energy mode emerges $\left[\omega / t_{\|} \approx 7\right.$ and $k_{\|} \approx \pi$ in Fig. 1(a)]. To clarify the nature of this mode, we consider an effective model for $U, t_{\perp} \gg t_{\|}$. In the low-electron-density regime, an electron with $k_{\perp}=0$ on a rung $\left|B_{\sigma}\right\rangle$ hops almost freely along the leg. When two electrons with opposite spins sit on the same rung, they are excited to one of the two-electron eigenstates with $k_{\perp}=0$ on a rung $\left|\psi^{ \pm}\right\rangle$(Table I). Thus, the effective model for $k_{\perp}=0$ can be obtained as the Hubbard chain with the following effective 

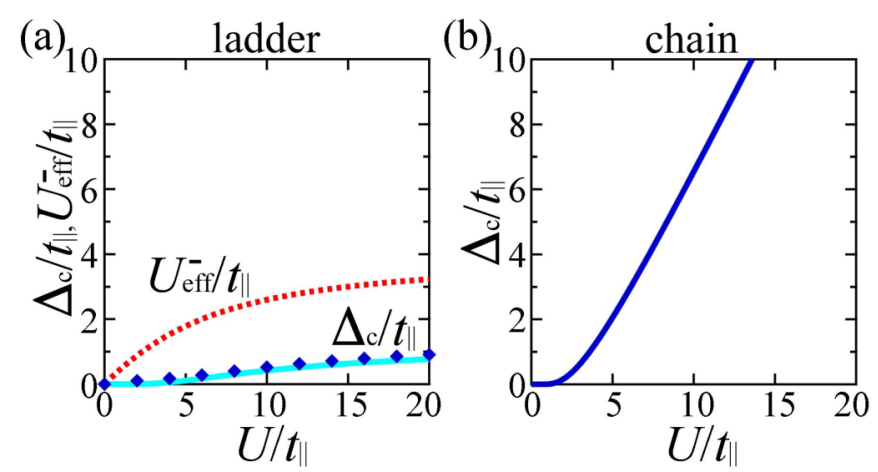

FIG. 4. (a) $U_{\text {eff }}^{-} / t_{\|}$for $t_{\perp} / t_{\|}=2$ [Eq. (14)] (dotted red curve). Charge gap $\Delta_{\mathrm{c}}$ of the Hubbard ladder at $n=1 / 2$ for $t_{\perp} / t_{\|}=2$ determined as the chemical-potential difference obtained using the non-Abelian DDMRG method in a 120 -site cluster (blue diamonds), and $\Delta_{\mathrm{c}}$ of the effective Hubbard chain with $U_{\text {eff }}^{-}$at $n_{\text {eff }}(=2 n)=1$ (solid cyan curve) obtained using (b). (b) $\Delta_{c}$ of the Hubbard chain at $n=1$ obtained using the Bethe ansatz [53].

interaction $U_{\mathrm{eff}}^{ \pm}[7]$ and effective chemical potential $\mu_{\mathrm{eff}}$ :

$$
U_{\text {eff }}^{ \pm}=E_{ \pm}+2 t_{\perp}, \quad \mu_{\text {eff }}=\mu+t_{\perp},
$$

by equating $E_{B}$ and $E_{\psi^{ \pm}}$with the effective single-site energies $-\mu_{\text {eff }}$ and $U_{\text {eff }}^{ \pm}-2 \mu_{\text {eff }}$, respectively.

In the Hubbard chain, the dispersion relation of the highenergy mode in a two-electron system can also be obtained from Eq. (6) as

$$
\epsilon_{k_{\|}}=U+4 t_{\|}+J_{\|}\left(\cos k_{\|}+1\right),
$$

up to $O\left(t_{\|}^{2} / U\right)$ in the large- $U / t_{\|}$regime $\left[t_{\perp}=J_{\perp}=0\right.$ in Eq. (7)]. By putting $U_{\text {eff }}^{ \pm}$[Eqs. (13) and (14)] into Eq. (15), we obtain

$$
\begin{aligned}
& \epsilon_{\left(k_{\|}, 0\right)}^{+}=U+2 t_{\perp}+J_{\perp}+4 t_{\|}+J_{\|}\left(\cos k_{\|}+1\right), \\
& \epsilon_{\left(k_{\|}, 0\right)}^{-}=2 t_{\perp}-J_{\perp}+4 t_{\|}+J_{\|}\left(\cos k_{\|}+1\right),
\end{aligned}
$$

up to $O\left(t_{\perp}^{2} / U\right)$ and $O\left(t_{\|}^{2} / U\right)$ for $U \gg t_{\perp} \gg t_{\|}$. The former $\left[\epsilon_{\left(k_{\|}, 0\right)}^{+}\right]$corresponds to the high-energy mode of $O(U)$ [Eqs. (7) and (11)]. The latter $\left[\epsilon_{\left(k_{\|}, 0\right)}^{-}\right]$corresponds to the emergent mode in the intermediate-energy regime $\left[\omega / t_{\|} \approx 7\right.$ and $k_{\|} \approx \pi$ in Fig. 1(a); dashed-dotted blue curve in Fig. 3(a)].

From the above analysis, the emergent mode in the intermediate-energy regime can be interpreted as the upper Hubbard band of the effective Hubbard chain with $U_{\text {eff }}^{-}=E_{-}+2 t_{\perp} \approx-J_{\perp}+2 t_{\perp}$ [dotted red curve in Fig. 4(a)]. Thus, the energy of this mode is not $O(U)$ but $O\left(t_{\perp}\right)$ for $U \gg t_{\perp} \gg t_{\|}$.

\section{Remarks on the upper Hubbard band}

In a conventional band picture, the splitting of a band into upper and lower bands is considered a result of symmetry breaking. For example, antiferromagnetic ordering, which causes folding of the Brillouin zone, is considered responsible for the formation of the gap in an antiferromagnetic insulator in a band picture. Nevertheless, the emergence of the highenergy states is a general characteristic of strongly interacting systems on a lattice, which does not require symmetry breaking or long-range order.

The mechanism of the emergence of the upper Hubbard band can, instead, be interpreted as the formation of a pair or a bound state [28-33]. The simplest case is the limit of the low-electron-density and strong repulsion where the high-energy mode can be interpreted as a mode of double occupancy (a bound state of electrons with opposite spins [28]) [Eq. (8)] as shown in Sec. V A. More generally, the interpretation as the formation of a pair can be justified in terms of string solutions in one-dimensional (1D) systems. Among Bethe-ansatz solutions, there are solutions involving a string which can be regarded as a pair of particles [34,35]. In the Hubbard chain, the upper Hubbard band has been identified as the $k-\Lambda$ string solutions [29]. Similarly, the highenergy states of the antiferromagnetic Heisenberg chain in a magnetic field in $S^{+-}(k, \omega)$ (excitation of flipping a majority spin to a minority spin) have been identified as the twostring solutions [30], which correspond to the upper Hubbard band of the interacting hard-core bosons [31] and interacting spinless fermions on a chain $[32,33]$. Thus, these high-energy states can be identified as states involving a pair of particles.

It should be noted that the quasiparticle responsible for the upper Hubbard band is not exactly the double occupancy, in general. In fact, the double occupancy exists even in the ground state at half-filling for $U<\infty$, and the double occupancy is not specified by a quantum number of eigenstates. In the Hubbard chain, the quasiparticle responsible for the upper Hubbard band has been identified in terms of the quantum number for the $k-\Lambda$ string [29] similarly to the spinon and holon (defined in terms of the quantum numbers for spin and charge, respectively). In higher dimensions, the quasiparticle responsible for the upper Hubbard band could be interpreted as a pair of particles [28] or that of a chain deformed by interchain hopping (Sec. V B) [36,37].

\section{QUARTER-FILLING}

At quarter-filling $(n=1 / 2)$, the system becomes a Mott insulator [Figs. 1(d), 1(1), and 2(d)] because the Mott transition occurs at $n_{\text {eff }}\left(=N_{\mathrm{e}} / L=2 n\right)=1$ in the effective Hubbard chain with $U_{\text {eff }}^{-}$for $k_{\perp}=0$ [Sec. V B].

To understand the nature of this dimer Mott insulator, we consider the electronic states of the upper Hubbard band of the effective Hubbard chain. As mentioned in Sec. V B, the two values of the effective $U, U_{\text {eff }}^{ \pm}=E_{ \pm}+2 t_{\perp}$ [Eq. (14)], reflect the two doubly occupied states on a rung (Table I),

$$
\begin{aligned}
& \left|\psi^{+}\right\rangle=-\zeta_{-}|S\rangle+\zeta_{+}\left|D^{+}\right\rangle, \\
& \left|\psi^{-}\right\rangle=\zeta_{+}|S\rangle+\zeta_{-}\left|D^{+}\right\rangle,
\end{aligned}
$$

where

$$
\zeta_{ \pm}=\sqrt{\frac{1}{2}\left(1 \pm \frac{U}{\sqrt{U^{2}+16 t_{\perp}^{2}}}\right)} .
$$

In the large- $U / t_{\perp}$ limit, $\left|\psi^{+}\right\rangle \rightarrow\left|D^{+}\right\rangle$and $\left|\psi^{-}\right\rangle \rightarrow|S\rangle$ because $\zeta_{+} \rightarrow 1$ and $\zeta_{-} \rightarrow 0$. Hence, the high-energy mode of $O(U)$ has a larger component of the doubly occupied sites $\left[\left|D^{+}\right\rangle=(|\uparrow \downarrow, 0\rangle+|0, \downarrow \uparrow\rangle) / \sqrt{2}\right]$, whereas the low-energy 
mode of $O\left(t_{\perp}\right)$ has a larger component of the spin-singlet state without doubly occupied sites $[|S\rangle=(|\uparrow, \downarrow\rangle-|\downarrow, \uparrow\rangle) /$ $\sqrt{2}]$ in the large- $U / t_{\perp}$ regime. Thus, in the dimer Mott insulator whose gap is essentially determined by $U_{\text {eff }}^{-}$, the doubly occupied state $\left|\psi^{-}\right\rangle$can primarily be regarded as the spin-singlet state without doubly occupied sites on a rung; the Mott gap is not of $O(U)$ but of $O\left(t_{\perp}\right)\left(U_{\text {eff }}^{-}=E_{-}+2 t_{\perp} \approx\right.$ $-J_{\perp}+2 t_{\perp}$ ) for $U \gg t_{\perp} \gg t_{\|}$[blue diamonds and solid cyan curve in Fig. 4(a)] [19].

The Mott gap and the effective $U$ are relevant not only to the charge excitation, but also to spin fluctuations and electronic excitations. The effective spin coupling at quarterfilling is $J_{\| \text {eff }}^{-}=4 t_{\|}^{2} / U_{\text {eff }}^{-}\left(\gg J_{\|}=4 t_{\|}^{2} / U\right)$ for $U \gg t_{\perp} \gg t_{\|}$. Hence, the spin degrees of freedom for $k_{\perp}=0$ can be described as the effective Heisenberg chain with $J_{\| \text {eff }}^{-}$, which shows a two-spinon continuum: $\omega=\frac{\pi J_{\text {leff }}^{-}}{2}\left(\sin p_{\|}^{1}+\sin p_{\|}^{2}\right)$, where $k_{\|}=p_{\|}^{1}+p_{\|}^{2}$ for $0 \leqslant p_{\|}^{1}<p_{\|}^{2} \leqslant \pi$, and the spin-wave mode at the lower edge: $\omega=\frac{\pi J_{\text {leff }}^{-}}{2}\left|\sin k_{\|}\right|$[38] [Fig. 2(b)].

By doping the dimer Mott insulator with a hole (an electron), the spin-wave mode emerges in the electronic-excitation spectrum with the dispersion relation shifted by the Fermi momenta $k_{\| \mathrm{F}}= \pm \pi / 2$ for $\omega>0(\omega<0)$ [Figs. 1(c), 1(e), 2(a), and 2(c)] as in the Hubbard chain [29]. The properties of the doping-induced states and the relationship to the Mott transition are discussed in detail in Sec. VIII.

It should be noted that the derivation of the effective $U, U_{\text {eff }}^{ \pm}$[Eq. (14)], is valid for $U, t_{\perp} \gg t_{\|}$(not only $U \gg$ $\left.t_{\perp} \gg t_{\|}\right)$for $n \leqslant 1 / 2$. In the case of $t_{\perp} \gg U \gg t_{\|}$,

$$
\begin{aligned}
& U_{\text {eff }}^{+}=4 t_{\perp}+\frac{U}{2}+\frac{U^{2}}{16 t_{\perp}}, \\
& U_{\text {eff }}^{-}=\frac{U}{2}-\frac{U^{2}}{16 t_{\perp}},
\end{aligned}
$$

up to $O\left(U^{2} / t_{\perp}\right)$. The dimer Mott gap determined by $U_{\text {eff }}^{-}$at $n_{\text {eff }}(=2 n)=1$ is of $O(U)$ for $t_{\perp} \gg U \gg t_{\|}$. Thus, in the case of $U, t_{\perp} \gg t_{\|}$, the dimer Mott gap at $n=1 / 2$ as well as $U_{\text {eff }}^{-}$ for $n \leqslant 1 / 2$ is limited by $2 t_{\perp}$ for $U \gg t_{\perp}$ [Fig. 4(a)] and by $U / 2$ for $t_{\perp} \gg U$ [Fig. 4(a) with $2 t_{\perp} \leftrightarrow U / 2$ ]. This implies that the effective Hubbard model with $U_{\text {eff }}>2 t_{\perp}\left(U_{\text {eff }}>U / 2\right)$ is not relevant to the low-energy properties of a dimer Mott insulator for $U \gg t_{\perp}\left(t_{\perp} \gg U\right)$. Because $U_{\text {eff }}^{ \pm}$is obtained in a dimer, the above argument would generally hold true for coupled-dimer systems $[6,7]$ regardless of the lattice structure or dimensionality as long as $U, t_{\perp} \gg t_{\|}$.

\section{HALF-FILLING}

\section{A. Electronic excitation}

At half-filling $(n=1)$, the ground state of the Hubbard ladder for $U \gg t_{\perp} \gg t_{\|}$can be effectively approximated as

$$
|\mathrm{GS}\rangle \approx \prod_{j=1}^{L}\left|\psi_{-}\right\rangle_{j} .
$$

The dominant modes excited from the ground state are obtained by replacing one of the $\left|\psi_{-}\right\rangle$'s with $|B\rangle,|A\rangle,|G\rangle$, and
$|F\rangle$ as

$$
\left|\overline{X\left(k_{\|}\right)}\right\rangle=\frac{1}{\sqrt{L}} \sum_{j=1}^{L} e^{i k_{\|} r_{j}}|X\rangle_{j} \prod_{l \neq j}^{L}\left|\psi_{-}\right\rangle_{l},
$$

where $X$ represents $B, A, G$, and $F$. The excitation energies up to the second order in $t_{\|}$are obtained as

$$
\epsilon_{k_{\|}}^{X}=-2 t_{\|}^{X} \cos k_{\|}+E_{X}-E_{\psi^{-}}+2 \xi_{X \psi^{-}}-2 \xi_{\psi^{-} \psi^{-}},
$$

where $E_{X}$ denotes the rung energy of $|X\rangle$ (Table I), and $t_{\|}^{B}=$ $-t_{\|}^{F}=t_{\|}^{+}$and $t_{\|}^{A}=-t_{\|}^{G}=t_{\|}^{-}$, where

$$
t_{\|}^{ \pm}=-\frac{t_{\|}}{2}\left(1 \pm \frac{4 t_{\perp}}{\sqrt{U^{2}+16 t_{\perp}^{2}}}\right) .
$$

Here, $\xi_{X \psi^{-}}$denotes the bond energy between $|X\rangle$ and $\left|\psi^{-}\right\rangle$ obtained in the second-order perturbation theory,

$$
\begin{aligned}
\xi_{\psi^{-} \psi^{-}}= & -\frac{2 t_{\|}^{2} U^{2}}{\left(U^{2}+16 t_{\perp}^{2}\right)^{3 / 2}}, \\
\xi_{T \psi^{-}}= & -\frac{4 t_{\|}^{2}}{U}+\frac{2 t_{\|}^{2}}{\sqrt{U^{2}+16 t_{\perp}^{2}}}, \\
\xi_{B \psi^{-}}= & -\frac{t_{\|}^{2}}{4 t_{\perp}}-\frac{t_{\|}^{2} \sqrt{U^{2}+16 t_{\perp}^{2}}}{8 t_{\perp} U}+\frac{\xi_{\psi^{-} \psi^{-}}}{8} \\
& +\frac{t_{\|}^{2}}{U}+\frac{t_{\|}^{2}}{\sqrt{U^{2}+16 t_{\perp}^{2}}}-\frac{2 t_{\|}^{2} t_{\perp}}{U \sqrt{U^{2}+16 t_{\perp}^{2}}}, \\
\xi_{A \psi^{-}}= & \left.\xi_{B \psi^{-}}\right|_{t_{\perp} \leftrightarrow-t_{\perp}}, \\
\xi_{G \psi^{-}}= & \xi_{A \psi^{-}}, \\
\xi_{F \psi^{-}}= & \xi_{B \psi^{-}},
\end{aligned}
$$

where $T$ represents $T^{+}, T^{-}$, or $T^{0}$.

We can also construct two-particle states by using $X(=$ $B, A, G$, and $F)$ and $Y\left(=T^{+}, T^{-}\right.$, or $\left.T^{0}\right)$ as

$$
\begin{aligned}
\left|\overline{X T\left(k_{\|} ; p_{\|}\right)}\right\rangle= & \frac{1}{\sqrt{L(L-1)}} \sum_{m \neq n} e^{i\left(k_{\|}-p_{\|}\right) r_{m}} e^{i p_{\|} r_{n}} \\
& \times|X\rangle_{m}|Y\rangle_{n} \prod_{l \neq m, n}^{L}\left|\psi_{-}\right\rangle_{l},
\end{aligned}
$$

whose effective excitation energies for $L \rightarrow \infty$ are obtained as

$$
\begin{aligned}
\epsilon_{k_{\|} ; p_{\|}}^{X T}= & -2 t_{\|}^{X} \cos \left(k_{\|}-p_{\|}\right)+J_{\|}^{\mathrm{eff}} \cos p_{\|}+E_{X}-2 E_{-} \\
& +2 \mu+2 \xi_{X \psi^{-}}+2 \xi_{T \psi^{-}}-4 \xi_{\psi^{-} \psi^{-}}
\end{aligned}
$$

where

$$
J_{\|}^{\mathrm{eff}}=\frac{8 t_{\|}^{2}}{U}-\frac{4 t_{\|}^{2}}{\sqrt{U^{2}+16 t_{\perp}^{2}}},
$$

which reduces to $J_{\|}^{\text {eff }} \approx J_{\|}$for $U \gg t_{\perp}$.

The dominant modes and the continua carrying considerable spectral weights in Figs. 1(h) and 1(p) can basically be identified with the above modes [Eqs. (21) and (22); dotted 

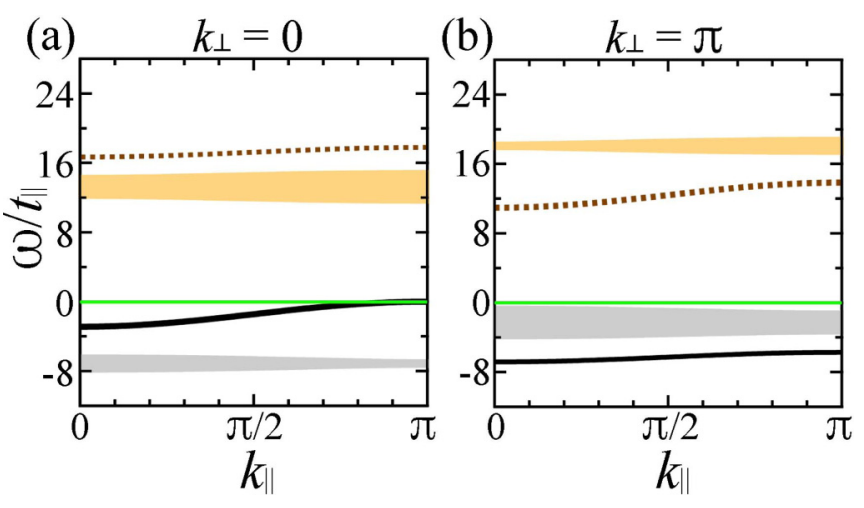

FIG. 5. Dispersion relation of electronic excitations at $n=1$ for $U / t_{\|}=16$ and $t_{\perp} / t_{\|}=2$ at [(a)] $k_{\perp}=0$ and [(b)] $\pi$. In (a), $\omega=\epsilon_{k_{\|}}^{G}$ (dotted brown curve), $-\epsilon_{k_{\|}}^{B}$ (solid black curve), $\epsilon_{k_{\|} ; p_{\|}}^{F T}$ (light orange region), and $-\epsilon_{k_{\|} ; p_{\|}}^{A T}$ (light gray region). In (b), $\omega=\epsilon_{k_{\|}}^{F}$ (dotted brown curve), $-\epsilon_{k_{\|}}^{A}$ (solid black curve), $\epsilon_{k_{\|} ; p_{\|}}^{G T}$ (light orange region), and $-\epsilon_{k_{\|} ; p_{\|}}^{B T}$ (light gray region). The green lines indicate $\omega=0$. The chemical potential $\mu$ is set so that $\epsilon_{\pi}^{B}=0$.

brown curves and solid black curves in Fig. 5] and the twoparticle states [Eqs. (25) and (26); light orange regions and light gray regions in Fig. 5].

\section{B. Spin excitation}

The spin excited state is similarly obtained as in Eq. (21) with $X=T^{+}, T^{-}$, or $T^{0}$, whose excitation energy up to the second order in $t_{\|}$can be obtained as

$$
\epsilon_{k_{\|}}^{\mathrm{spin}}=J_{\|}^{\mathrm{eff}} \cos k_{\|}-E_{-}+2 \xi_{T \psi^{-}}-2 \xi_{\psi^{-} \psi^{-}} .
$$

This excitation well explains the mode in $S(\boldsymbol{k}, \omega)$ for $k_{\perp}=\pi$ at half-filling [Fig. 6(b)].

\section{MOTT TRANSITION}

\section{A. Doping-induced states}

The most remarkable spectral feature is the emergence of electronic states in the Mott gap by doping a Mott insulator
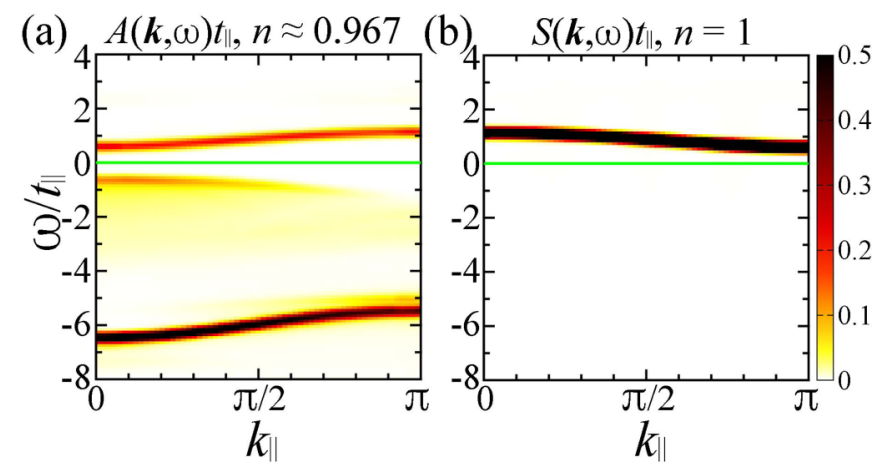

FIG. 6. (a) $A(\boldsymbol{k}, \omega) t_{\|}$for $k_{\perp}=\pi$ at $n \approx 0.967$ for $U / t_{\|}=16$ and $t_{\perp} / t_{\|}=2$ obtained using the non-Abelian DDMRG method [closeup of Fig. 1(o) near the Fermi level]. (b) $S(\boldsymbol{k}, \omega) t_{\|}$for $k_{\perp}=$ $\pi$ at $n=1$ for $U / t_{\|}=16$ and $t_{\perp} / t_{\|}=2$ obtained using the nonAbelian DDMRG method. The green lines indicate $\omega=0$. Gaussian broadening with a standard deviation of $0.1 t_{\|}$has been used.
[Figs. 1(o) and 1(p)]. From the low-electron-density side, the dominant mode for $k_{\perp}=\pi$ in the low-electron-density regime loses spectral weight and disappears at half-filling [Figs. 1(i)-1(p)], but its dispersion relation remains dispersing until the Mott transition occurs [Figs. 1(o) and 6(a)]. In the small-doping limit, the dispersion relation reduces to the magnetic dispersion relation shifted by the Fermi momentum $\boldsymbol{k}_{\mathrm{F}}=(\pi, 0)$ (Fig. 6). Thus, the antibonding band in the lowelectron-density regime gradually loses spectral weight with the electron density and eventually leads to the magnetic excitation at half-filling. This implies that the charge degrees of freedom freeze, whereas the spin degrees of freedom remain active toward the Mott transition [5,27,29,36].

From the Mott-insulator side, this feature can be described as follows: the spin excited states at half-filling appear in the electron-addition spectrum with the dispersion relation shifted by the Fermi momentum upon doping a Mott insulator because the charge characteristic is added to the spin excited states by doping [5,27,29,36]. A simple explanation for this feature has been given using effective eigenstates of the $t-J$ ladder as well as based on general arguments on quantum numbers in Ref. [27]. Here, we briefly review the explanation in the case of the Hubbard ladder. The ground state at halffilling for $U \gg t_{\perp} \gg t_{\|}$can be effectively approximated as in Eq. (20). The spin excited states are obtained as $\left|\overline{T\left(k_{\|}\right)}\right\rangle(T=$ $T^{+}, T^{-}$, or $T^{0}$ ) [Eq. (21)] whose dispersion relation is expressed as $\omega=\epsilon_{k_{\|}}^{\text {spin }}$ [Eq. (28); Fig. 6(b)]. The one-hole-doped ground state is essentially $|\overline{B(\pi)}\rangle$, which has momentum $\boldsymbol{k}=$ $(\pi, 0)$. In the small-doping limit, the chemical potential is set so that the energy of the one-hole-doped ground state is zero. When an electron with momentum $\left(p_{\|}, \pi\right)$ is added to the one-hole-doped ground state, the obtained state has overlap with the spin excited state at half-filling $\left|\overline{T\left(p_{\|}+\pi\right)}\right\rangle$. Thus, $A\left(\left(p_{\|}, \pi\right), \omega\right)$ exhibits a mode along $\omega=\epsilon_{p_{\|}+\pi}^{\text {spin }}$ [Eq. (28); Fig. 6(a)]. This argument shows that the spin excited states at half-filling appear in the electron-addition spectrum with the dispersion relation shifted by the Fermi momentum upon doping a Mott insulator.

This feature of the doping-induced states has also been pointed out in the Hubbard chain [29], two-dimensional (2D) Hubbard model [36], $t-J$ chain [39], 2D $t-J$ model [26], and $t-J$ ladder [27], as well as in a system with antiferromagnetic order [40]. In the Hubbard ladder considered in this paper, the mode of the doping-induced sates has an energy gap because the spin excitation at half-filling has an energy gap (Fig. 6). In the case where the spin excitation is gapless in a Mott insulator, the mode of the doping-induced states should be gapless, as shown in Fig. 2, and in the Hubbard and $t-J$ chains, and the 2D Hubbard and $t-J$ models [5,26,27,29,36,39-41].

The emergence of electronic states upon doping a Mott insulator was recognized soon after the discovery of cuprate high-temperature superconductors $[3,42,43]$. However, interpretations are controversial. Various interpretations other than the above interpretation have been proposed primarily for the 2D Hubbard model [44-48], suggesting that the mode of the doping-induced states is essentially separated by a (pseudo-)gap from the low-energy band even though the Mott insulator exhibits gapless spin excitation [44-48]. In contrast, the interpretation described above as well as in 
Refs. [5,26,27,29,36,39-41] can naturally and collectively explain the behavior of the doping-induced states in the Hubbard and $t-J$ chains [29,39] and the Hubbard and $t-J$ ladders [27] (Figs. 2 and 6) as well as in the 2D Hubbard and $t-J$ models $[5,26,36]$, which implies that this interpretation captures the essence of the Mott transition.

\section{B. What characterizes the Mott transition}

To discuss how to characterize the Mott transition, the definition of the Mott transition must be clarified. In particular, the definition should be what can distinguish the Mott transition from the transition between a metal and a band insulator. Hence, a clear distinction between a Mott insulator and a band insulator is required.

One might consider that a Mott insulator could be defined by the value of the charge gap; if the charge gap is primarily determined by the Coulomb repulsion $[O(U)$ in the Hubbard model], the insulating state could be regarded as a Mott insulator. However, as shown in Sec. VI, even though the value of the charge gap is limited by the intradimer hopping for $U \gg t_{\perp} \gg t_{\|}$, the insulating state at quarter-filling should be regarded as a Mott insulator because it is essentially the same as the Mott insulator of the Hubbard chain (Fig. 2) [Sec. VI]. Thus, a Mott insulator is not necessarily well-defined in terms of the value of the charge gap.

Another definition could be that a Mott insulator is an insulator exhibiting gapless spin excitation. Such an insulator is not a band insulator because the spin gap is basically equal to the charge gap in a band insulator. However, this definition appears too narrow; in practice, a system having low-energy spin excitation with a large charge gap is generally called a Mott insulator regardless of whether a small spin gap opens or not. Hence, a Mott insulator can be better defined as an insulator with $\Delta_{\mathrm{s}} \ll \Delta_{\mathrm{c}}$ (or $\Delta_{\mathrm{s}}<\Delta_{\mathrm{c}}$ if necessary), where $\Delta_{\mathrm{S}}$ and $\Delta_{\mathrm{c}}$ denote the lowest excitation energies for spin and charge, respectively. This implies that a Mott insulator can be defined in terms of the spin-charge separation $\left(\Delta_{\mathrm{s}} \neq \Delta_{\mathrm{c}}\right)$ [27]. In 1D systems, the spin-charge separation is considered to occur even in a metallic phase: The properties in the low-energy limit are described in terms of spin and charge excitations independent of each other with different velocities rather than electronlike quasiparticles [49-52]. In a Mott insulator, regardless of the lattice structure or dimensionality, the spin-charge separation $\left(\Delta_{\mathrm{s}} \ll \Delta_{\mathrm{c}}\right)$ occurs more clearly than in a $1 \mathrm{D}$ metal $\left[\Delta_{\mathrm{s}} \neq \Delta_{\mathrm{c}}=O(1 / L)\right]$.

If a Mott insulator is characterized by $\Delta_{\mathrm{s}} \ll \Delta_{\mathrm{c}}$, what characterizes the Mott transition should also reflect it. If only the ground-state properties are considered, the Mott transition in a dimerized system, such as the Hubbard ladder for $U \gg t_{\perp} \gg t_{\|}$at half-filling, is essentially the same as the transition to a band insulator [Figs. 1(g) and 1(h)]. However, reflecting $\Delta_{\mathrm{s}} \ll \Delta_{\mathrm{c}}$ of the Mott insulator [Figs. 1(h), $1(\mathrm{p})$, and 6(b)], electronic states exhibiting the momentumshifted magnetic dispersion relation emerge in the Mott gap by doping [Figs. 1(o) and 6(a)]. This characteristic of the Mott transition reflects the characteristic of the Mott insulator (spin-charge separation: $\Delta_{\mathrm{s}} \ll \Delta_{\mathrm{c}}$ ) and does not appear in the transition from a band insulator. Hence, this characteristic should be general and fundamental to the Mott transition [5,26,27,29,36,39-41]. The above argument implies that the Mott transition is characterized by the doping-induced states that exhibit the momentum-shifted magnetic dispersion relation rather than critical exponents or order parameters.

\section{SUMMARY}

The emergence, disappearance, and spectral-weight transfer of electronic states are illustrated in the Hubbard ladder in the strong repulsion and strong intrarung hopping regime. The dominant modes in the low-electron-density regime significantly lose spectral weight as the electron density increases to half-filling, whereas the emergent modes in the low-electrondensity regime become dominant at half-filling; the dominant modes in the low-electron-density regime and those at halffilling are different in origin.

One of the emergent modes, which has an energy of the order of the intradimer hopping, significantly gains spectral weight and governs the dimer Mott physics at quarter-filling; the dimer Mott gap is limited by the intradimer hopping even in the strong repulsion regime.

In contrast, one of the dominant modes in the low-electrondensity regime, which originates from a noninteracting band, gradually loses spectral weight as the electron density increases, and completely disappears at half-filling. However, the dispersion relation remains dispersing until the Mott transition occurs; the dispersion relation reduces to the magnetic dispersion relation shifted by the Fermi momentum in the small-doping limit. Thus, the mode originating from a noninteracting band continuously leads to the mode of the spin excitation at half-filling.

These features would be basically true for general coupleddimer systems regardless of the lattice structure or dimensionality as long as $U \gg t_{\perp} \gg t_{\|}$. As the dimer Mott gap is limited by the intradimer hopping, a Mott insulator is not necessarily well-defined as an insulator having a charge gap of the order of the Coulomb repulsion; a Mott insulator is better characterized in terms of the existence of spin excitation whose energy is much lower than the charge gap, i.e., the spin-charge separation. By reflecting this characteristic of a Mott insulator, the Mott transition can be characterized: The spin excited states in a Mott insulator emerge in the Mott gap as electronic excitation by doping the Mott insulator, exhibiting the momentum-shifted magnetic dispersion relation. This feature does not appear in the transition from a band insulator and should be general and fundamental to the Mott transition.

In the small- $t_{\perp} / t_{\|}$regime, the spectral-weight distribution can be affected by band hybridization. Nevertheless, the overall spectral features such as emergence and disappearance of spectral weight should generally appear in strongly correlated systems.

The emergence, disappearance, and spectral-weight transfer, which have almost been overlooked in band theory and Fermi-liquid theory, play particularly important roles in understanding the physics around the Mott transition, such as the origin of the Mott gap and the doping-induced states. This would be one of the reasons why the electronic properties near the Mott transition have appeared elusive from the 
conventional viewpoints. This paper also brings an unconventional perspective to electronic bands. In a conventional band picture, electronic bands are usually identified as those primarily originating from atomic orbitals; the number of bands is considered invariant regardless of the electron density as long as symmetry breaking does not occur. However, as shown in this paper, a noninteracting band at zero electron density, which corresponds to a conventional band, can disappear at the Mott transition, whereas emergent electronic bands in the low-electron-density regime can become dominant near the Mott transition. The number of electronic bands carrying significant spectral weight can vary depending on the electron density even without symmetry breaking in strongly correlated systems. Experimental confirmation of these features over a wide energy and electron-density regime as well as applications of the emergence of electronic states to electronic or optical devices is desired.

\section{ACKNOWLEDGMENTS}

The author would like to thank S. Uji and S. Tsuda for helpful discussions. This work was supported by JSPS KAKENHI Grant No. JP26400372 and the JST-Mirai Program Grant No. JPMJMI18A3, Japan. The numerical calculations were partly performed on the supercomputer at the National Institute for Materials Science.
[1] N. W. Ashcroft and N. D. Mermin, Solid State Physics (Cengage Learning, Boston, 1976).

[2] P. Nozières, Theory of Interacting Fermi Systems (W. A. Benjamin, New York, 1964).

[3] E. Dagotto, Correlated electrons in high-temperature superconductors, Rev. Mod. Phys. 66, 763 (1994).

[4] M. Imada, A. Fujimori, and Y. Tokura, Metal-insulator transitions, Rev. Mod. Phys. 70, 1039 (1998).

[5] M. Kohno, Characteristics of the Mott transition and electronic states of high-temperature cuprate superconductors from the perspective of the Hubbard model, Rep. Prog. Phys. 81, 042501 (2018).

[6] H. Seo, C. Hotta, and H. Fukuyama, Toward systematic understanding of diversity of electronic properties in low-dimensional molecular solids, Chem. Rev. 104, 5005 (2004).

[7] H. Kino and H. Fukuyama, Electronic states of conducting organic $\kappa$-(BEDT-TTF) $)_{2}$ X, J. Phys. Soc. Jpn. 64, 2726 (1995).

[8] L. Balents and M. P. A. Fisher, Weak-coupling phase diagram of the two-chain Hubbard model, Phys. Rev. B 53, 12133 (1996).

[9] R. M. Noack, S. R. White, and D. J. Scalapino, Correlations in a Two-Chain Hubbard Model, Phys. Rev. Lett. 73, 882 (1994).

[10] R. M. Noack, N. Bulut, D. J. Scalapino, and M. G. Zacher, Enhanced $d_{x^{2}-y^{2}}$ pairing correlations in the two-leg Hubbard ladder, Phys. Rev. B 56, 7162 (1997).

[11] M. Dolfi, B. Bauer, S. Keller, and M. Troyer, Pair correlations in doped Hubbard ladders, Phys. Rev. B 92, 195139 (2015).

[12] S. Liu, H.-C. Jiang, and T. P. Devereaux, Nature of a single doped hole in two-leg Hubbard and $t-J$ ladders, Phys. Rev. B 94, 155149 (2016).

[13] A. Nocera, Y. Wang, N. D. Patel, G. Alvarez, T. A. Maier, E. Dagotto, and S. Johnston, Doping evolution of charge and spin excitations in two-leg Hubbard ladders: Comparing DMRG and FLEX results, Phys. Rev. B 97, 195156 (2018).

[14] Z. Weihong, J. Oitmaa, C. J. Hamer, and R. J. Bursill, Numerical studies of the two-leg Hubbard ladder, J. Phys.: Condens. Matter 13, 433 (2001).

[15] H. Endres, R. M. Noack, W. Hanke, D. Poilblanc, and D. J. Scalapino, Dynamical properties of two coupled Hubbard chains at half-filling, Phys. Rev. B 53, 5530 (1996).
[16] C. Yang and A. E. Feiguin, Spectral function of Mott-insulating Hubbard ladders: From fractionalized excitations to coherent quasiparticles, Phys. Rev. B 99, 235117 (2019).

[17] L. G. G. V. Dias da Silva, G. Alvarez, and E. Dagotto, Dynamics of doublon-holon pairs in Hubbard two-leg ladders, Phys. Rev. B 86, 195103 (2012).

[18] H. Hashimoto and S. Ishihara, Photoinduced correlated electron dynamics in a two-leg ladder Hubbard system, Phys. Rev. B 93, 165133 (2016).

[19] M. Kohno, Aspects of the ground state of the $U=\infty$ Hubbard ladder, Phys. Rev. B 56, 15015 (1997).

[20] V. Ya. Krivnov, A. A. Ovchinnikov, and V. O. Cheranovskii, Magnetic properties of the hubbard model with infinite interactions, Synth. Met. 33, 65 (1989).

[21] L. Liu, H. Yao, E. Berg, S. R. White, and S. A. Kivelson, Phases of the Infinite $U$ Hubbard Model on Square Lattices, Phys. Rev. Lett. 108, 126406 (2012).

[22] E. Jeckelmann, Dynamical density-matrix renormalizationgroup method, Phys. Rev. B 66, 045114 (2002).

[23] I. P. McCulloch and M. Gulácsi, The non-Abelian density matrix renormalization group algorithm, Europhys. Lett. 57, 852 (2002).

[24] I. P. McCulloch, A. R. Bishop, and M. Gulacsi, Density matrix renormalization group algorithm and the two-dimensional t-J model, Philos. Mag. B 81, 1603 (2001).

[25] I. P. McCulloch, Collective Phenomena in Strongly Correlated Electron Systems, Ph.D. thesis, Australian National University, 2001).

[26] M. Kohno, Spectral properties near the Mott transition in the two-dimensional $t-J$ model, Phys. Rev. B 92, 085128 (2015).

[27] M. Kohno, States induced in the single-particle spectrum by doping a Mott insulator, Phys. Rev. B 92, 085129 (2015).

[28] J. Hubbard, Electron correlations in narrow energy bands, Proc. R. Soc. London, Ser. A 276, 238 (1963).

[29] M. Kohno, Spectral Properties near the Mott Transition in the One-Dimensional Hubbard Model, Phys. Rev. Lett. 105, 106402 (2010).

[30] M. Kohno, Dynamically Dominant Excitations of String Solutions in the Spin-1/2 Antiferromagnetic Heisenberg Chain in a Magnetic Field, Phys. Rev. Lett. 102, 037203 (2009). 
[31] M. Kohno, Relation between high-energy quasiparticles of quasi-one-dimensional antiferromagnets in a magnetic field and a doublon of a Hubbard chain, J. Phys.: Conf. Ser. 320, 012021 (2011).

[32] M. Kohno, M. Arikawa, J. Sato, and K. Sakai, Spectral Properties of Interacting One-Dimensional Spinless Fermions, J. Phys. Soc. Jpn. 79, 043707 (2010).

[33] R. G. Pereira, S. R. White, and I. Affleck, Spectral function of spinless fermions on a one-dimensional lattice, Phys. Rev. B 79, 165113 (2009).

[34] M. Takahashi, One-Dimensional Hubbard Model at Finite Temperature, Prog. Theor. Phys. 47, 69 (1972).

[35] H. Bethe, Zur Theorie der Metalle, Z. Phys. 71, 205 (1931).

[36] M. Kohno, Mott Transition in the Two-Dimensional Hubbard Model, Phys. Rev. Lett. 108, 076401 (2012).

[37] M. Kohno, Quasiparticles of Spatially Anisotropic Triangular Antiferromagnets in a Magnetic Field, Phys. Rev. Lett. 103, 197203 (2009).

[38] J. des Cloizeaux and J. J. Pearson, Spin-wave spectrum of the antiferromagnetic linear chain, Phys. Rev. 128, 2131 (1962).

[39] M. Kohno, Doping-induced states in the single-particle spectrum originating from magnetic excitation of a Mott insulator, Phys. Procedia 75, 206 (2015).

[40] M. Kohno, Doping-induced states near the Mott transition in the presence of antiferromagnetic order, AIP Adv. 8, 101302 (2018).

[41] M. Kohno, Relationship between single-particle excitation and spin excitation at the Mott transition, JPS Conf. Proc. 3, 013020 (2014).

[42] H. Eskes, M. B. J. Meinders, and G. A. Sawatzky, Anomalous Transfer of Spectral Weight in Doped Strongly Correlated Systems, Phys. Rev. Lett. 67, 1035 (1991).
[43] E. Dagotto, A. Moreo, F. Ortolani, J. Riera, and D. J. Scalapino, Density of States of Doped Hubbard Clusters, Phys. Rev. Lett. 67, 1918 (1991).

[44] S. Sakai, Y. Motome, and M. Imada, Evolution of Electronic Structure of Doped Mott Insulators: Reconstruction of Poles and Zeros of Green's Function, Phys. Rev. Lett. 102, 056404 (2009).

[45] Y. Yamaji and M. Imada, Composite-Fermion Theory for Pseudogap, Fermi Arc, Hole Pocket, and Non-Fermi Liquid of Underdoped Cuprate Superconductors, Phys. Rev. Lett. 106, 016404 (2011).

[46] P. Phillips, T.-P. Choy, and R. G. Leigh, Mottness in hightemperature copper-oxide superconductors, Rep. Prog. Phys. 72, 036501 (2009).

[47] R. Eder, K. Seki, and Y. Ohta, Self-energy and Fermi surface of the two-dimensional Hubbard model, Phys. Rev. B 83, 205137 (2011).

[48] R. Eder and Y. Ohta, Inverse photoemission in strongly correlated electron systems, Phys. Rev. B 54, 3576 (1996).

[49] F. D. M. Haldane, 'Luttinger liquid theory' of one-dimensional quantum fluids. I. Properties of the Luttinger model and their extension to the general 1D interacting spinless Fermi gas, J. Phys. C 14, 2585 (1981).

[50] S.-i. Tomonaga, Remarks on Bloch's method of sound waves applied to many-fermion problems, Prog. Theor. Phys. 5, 544 (1950).

[51] J. M. Luttinger, An exactly soluble model of a many-fermion system, J. Math. Phys. 4, 1154 (1963).

[52] D. C. Mattis and E. H. Lieb, Exact solution of a many-fermion system and its associated boson field, J. Math. Phys. 6, 304 (1965).

[53] E. H. Lieb and F. Y. Wu, Absence of Mott Transition in an Exact Solution of the Short-Range, One-Band Model in One Dimension, Phys. Rev. Lett. 20, 1445 (1968). 